Article

\title{
Simplicity and Sustainability: Pointers from Ethics and Science
}

\author{
Mehrdad Massoudi ${ }^{1, *}$ and Ashwin Vaidya ${ }^{2}$ \\ 1 Center for Nonlinear Analysis, Department of Mathematical Sciences, Carnegie Mellon University, \\ Pittsburgh, PA 15213-3890, USA \\ 2 Department of Mathematical Sciences, Montclair State University, Montclair, NJ 07043, USA; \\ vaidyaa@mail.montclair.edu \\ * Correspondence: massoudi@cmu.edu; Tel.: +1-412-854-4753
}

Received: 14 December 2017; Accepted: 19 April 2018; Published: 23 April 2018

\begin{abstract}
In this paper, we explore the notion of simplicity. We use definitions of simplicity proposed by philosophers, scientists, and economists. In an age when the rapidly growing human population faces an equally rapidly declining energy/material resources, there is an urgent need to consider various notions of simplicity, collective and individual, which we believe to be a sensible path to restore our planet to a reasonable state of health. Following the logic of mathematicians and physicists, we suggest that simplicity can be related to sustainability. Our efforts must therefore not be spent so much in pursuit of growth but in achieving a sustainable life.
\end{abstract}

Keywords: sustainability; simplicity; ethics; entropy; mathematical modeling

\section{Introduction}

Rapidly growing human population coupled with an increased rate of consumption, catalyzed by scientific and technological revolutions, have resulted in complex human societies. The march towards modernity is characterized by upholding certain social developments and measures, which are often characterized by very quantitative and tangible measures such as the Gross Domestic Product (GDP), UNDP's Human Development Index (HDI), the Index of Sustainable Economic Welfare (ISEW), the Genuine Progress Indicator (GPI), Environmentally Sustainable National Income (eSNI), Sustainable Development Indicators (SDI), National Accounts of Well-being (NAW), Calvert-Henderson Index, and others [1]. In more recent times, there have been attempts to convert intangible elements of success and progress into more tangible ones such as the happiness index [2]. However, these are few and do not resonate with many human beings around the world, as much as the economic indicators. The "value" of intangible elements such as quality, simplicity, satisfaction, wisdom, happiness, etc. have certainly diminished [3] over time. In 2015, the United Nations laid out several goals as a path to achieve sustainable development across the planet. These included 17 sub-goals each of which would be a tremendous achievement for humanity, among which are protection of the planet, ending poverty and achieving a more equitable distribution of wealth [4].

In this article, we examine a particular quality which we believe to be central to the notion of sustainability-simplicity. Understanding the true meaning of these intangibles is important to the sustenance and longevity of the human race and all other species. In this paper, we consider a few definitions of "simplicity" from diverse perspectives ranging from the wisdom traditions to modern scientific ones and highlight their convergent meanings and implications. The central objective of our paper lies in clarifying the role of simplicity in achieving some of these goals of sustainability proposed by the UN. Simplicity is a complex word rich in meaning, but is rarely discussed in the mainstream, even by academics who have become more fascinated with theories of complexity [5]. 
In Section 2, we build the foundation of this paper by providing some background information and show the interconnections between systems, energy, entropy and ethics. In Section 3, we show how simple ideas in mathematical modeling can be used to help us understand the economics of population growth. In Section 4, we discuss various aspects of simplicity. This section presents the core ideas in the paper. In Section 5, we attempt to relate simplicity to sustainability using simple mathematical ideas. In Section 6, we provide a few concluding remarks and end the paper with suggesting two environmental norms (EN).

\section{Systems, Energy, and Entropy}

To live sustainably on earth requires knowledge of the earth (environment), our behavior and actions and their consequences on the environment. Self-knowledge is the prerequisite here. By self-knowledge, we mean knowing ourselves: what our world-view is, what we believe, how we came to believe what we believe, what we think we should do, the meaning of our lives, etc. There is, of course, the scientific aspect of knowledge about earth: studying the weather patterns, the growth or decay/disappearance of species and plants, the rise and fall of the sea levels, the formation of rocks, etc. To study energy-related issues and their impact on environment, we need to discuss systems and thermodynamics.

Systems Theory is a general theory dealing with connected parts. A system, generally, consists of three parts, related to each other: elements, interconnections between the elements, and a function (or purpose). As explained by Meadows [6] (p. 2): "A system is a set of things-people, cells, molecules or whatever-interconnected in such a way that they produce their own patterns of behavior over time". There are many sub-subsystems in a given system. As Laszlo [7] (p. 140) says: "A system in one perspective is a subsystem in another. But the systems view always treats systems as integrated wholes of their subsidiary components and never as the mechanistic aggregate of parts in isolable causal relations". In a sense, a systems view is related to a (w)holistic view, where not only the parts (the sub-systems) of a system can be studied, but also the inter-connections between the parts and the relations between this system as a whole with its outside environment (another system). Macy [8] (p. 76) summarizes the four-fold properties of a system:

- $\quad$ "The system is a nonsummative whole, that is, it cannot be reduced to its parts without altering their pattern. Aggregates, like a brick wall or a library or the content of a shopping cart, where components can be added or subtracted without affecting the relations between other components, do not qualify.

- The system is homeostatic. It stabilizes itself through negative feedback; that is, the system adjusts its output to produce and sustain a match between the input it will receive and its internally coded requirements.

- The system is self-organizing. Where a mismatch between input and code persists, the system searches for and encodes a new pattern by which it can function. Incorporating positive feedback, differentiation and complexification of structure emerge.

- The system is not only a whole, but part within a larger whole. Whether a cell or organ, atom or animal, it comprises subsystems. It also is, itself, a subsystem within a wider system of whose character and in whose functioning it is an integral and codeterminative component. Open systems in interaction form more inclusive structures or patterns as a function of their mutual adaptations".

The distinction between a fragmentary view and a partial view of something is also crucial to our understanding of systems and their interactions. A partial view may eventually lead, under proper conditions, to a holistic view. A fragmentary view will not. Bohm [9] (p. 23) talks about this issue:

"A part, as I said-whether mechanical or organic-is intrinsically related to the whole, but this is not so for a fragment. As the Latin root of the word indicates, and as the related English word 'fragile' shows, to fragment is to break up or smash. To hit a watch with a hammer would not produce parts, but fragments that are separated in ways that are not significantly related to the structure of the watch". 
A convenient way of connecting the systems theory to energy-related problems and their impact on the environment is through the (classical) thermodynamics [10], where three types of systems are discussed [11]:

1. An isolated system: No exchange of energy or matter with the outside environment.

2. A closed system: Only energy is exchanged with the outside environment. Earth is an example of such a system. Interestingly, as mentioned in Moran and Shapiro [12] (p. 2), in a closed system, the boundaries of the system do not play a key role; instead, it is the quantity of matter which is being studied.

3. An open system: Both matter and energy can be exchanged with the outside environment.

In a closed system such as the earth, we can have an isolated sub-system, a closed sub-system, and an open sub-system; these subsystems can move (or can be transformed) from one state to another state. The process can be reversible (implying frictionless processes, i.e., no dissipation) or irreversible [13]. A state may be in equilibrium or disequilibrium, stable or unstable [13]. As von Bertalanffy [14] (p. 215) says:

"Any system is an entity which can be investigated in its own right must have boundaries, either spatial or dynamic. Strictly speaking, spatial boundaries exist only in naïve observations, and all boundaries are ultimately dynamic. One cannot exactly draw the boundaries of an atom (with valences sticking out, as it were, to attract other atoms), of a stone (an aggregate of molecules and atoms which mostly consist of empty space, with particles in planetary distances), or of an organism (continually exchanging matter with the environment)".

Much of the classical thermodynamics is based on the four fundamental laws of thermodynamics [15]. The Zeroth law states that Kestin [15] (p. 40): "Two systems in thermal equilibrium with a third system are in thermal equilibrium with each other". According to Kestin and Dorfan [16] (p. 15): "The Third law asserts that the entropy difference in an isothermal process tends to zero as the thermodynamic temperature tends to zero". The first and second laws are the ones more directly related to environmental issues, especially sustainability. The first law of thermodynamics, also known as the law of conservation of energy, states that "all matter and energy in the universe is constant, that it cannot be created or destroyed" [17]. The first law of thermodynamics does not say anything about the direction of this change. The second law of thermodynamics, or the Entropy law, in the context of classical thermodynamics is related to the free energy of a system. Lindsay [18] says: "If the essence of the first principle in everyday life is that we cannot get something for nothing, the second principle emphasizes that every time we do get something we reduce by a measurable amount the opportunity to get that something in the future, until ultimately the time will come when there will be no more getting". There are two well-known statements of the second law: one emphasizing the efficiency of conversion of heat into work, and the other the irreversibility of nature [9]:

"No process is possible whose sole result is the complete conversion of heat into work". [Kelvin]

"No process is possible whose sole result is the transfer of heat from a colder to a hotter body". [Clausius]

The entropy law has been applied in many different situations, such as ecology, economics, etc. [19]. Massoudi [20] extended the ideas proposed by Lindsay [18] and stated ethical imperatives based on the entropy law; the two thermodynamic imperatives (TI) discussed in [20] are:

TI.1 “We ought to do things, in so far as possible, in such ways that the production of entropy is minimized [20]".

TI.2 "We ought to do things, in so far as possible, in such ways that the consumption of entropy is maximized [18]". 
The first statement is concerned with conservation, simplicity, and harmony, while the second imperative considers ordering, sorting, and organizing. It is primarily through the application of TI.1 that, on an individual level, one can make ethical choices which when applied to nature could lead to conservation measures, and when applied to the choices that we make in life, could lead to a simpler and more harmonious life. In the following section, we provide a summary of the systems theory and its possible implications on the environment using dynamical systems modeling. The power of simple mathematical thinking in elucidating complex phenomena is also discussed. The ideas in the mathematical modeling section can then be used to motivate the following sections about understanding simplicity from an abstract point of view and using it to strive towards achieving sustainability.

\section{Mathematical Modeling}

(Mathematical) Modeling is an attempt or a desire to formulate, using mathematical symbols and ideas, relationships among seemingly unrelated phenomena. In mathematical modeling, we try to explain something, and, in some cases, to predict the behavior or response of something. It is possible to have different models attempting to explain the same phenomenon. Mathematical modeling is an idealization of some aspect of nature and represents an aspect of nature; it can be applied to physical or chemical processes, to economics, environment, etc. Aris [21] (p. 1) says: “... the term 'mathematical model' $^{\prime} .$. will be used for any complete and consistent set of mathematical equations which is thought to correspond to some other entity, its prototype. The prototype may be a physical, biological, social, psychological or conceptual entity, perhaps even another mathematical model ..." These mathematical relationships can be based on (see [21] (p. 26)) mechanical analogies (such as springs or dashpots), finite models (such as theory of games), fuzzy subsets, statistical models, stochastic models, models involving differential equations, etc. It is the last category that we use in this paper to illustrate the idea of sustainability. Truesdell [22] (p. 30) says: "Any mathematical theory of physics must idealize nature. ... In a sense, then, every theory is only 'approximate' in respect to nature itself. This unavoidable defect in theory is often taken as a patent for 'approximate' mathematics in deductions from it".

The mathematical modeling of population versus resources [23] is a relevant example and useful in our discussion and it will shed some light on this issue from a more tangible quantitative perspective while appealing also to the ethical and practical problem. It is well known that the population of a species depends strongly upon the carrying capacity of its environment, as reflected by the simple first order differential equation, typically denoted by [23]

$$
d P / d t=r P(1-P / K)
$$

where $t$ is the time, $P(t)$ is the time dependent population, $r$ is the population growth rate and $K$ denotes the carrying capacity of the environment, which is assumed to be constant. Assuming an initial population $P_{0}$, the general solution to this equation is given by

$$
\begin{gathered}
P(t)=\frac{K}{1+a e^{r t}} \\
a=\frac{\left(K-P_{0}\right)}{P_{0}}
\end{gathered}
$$

The graph of this equation for $P(t)$ is shown in Figure 1a for two different cases of initial population: (i) $P_{0}>K$; and (ii) $P_{0}<K$. Both situations indicate that the best possible final state is determined by the constant carrying capacity of the environment. In fact, the blue curve is very revealing; if the initial population is greater than the limits set by the environment, the excess population must die out until there is just enough to be sustained by the environment.

The saturating profile of the function $P(t)$ is not completely realistic since the carrying capacity of the environment is not necessarily a constant. Indeed, when the population is very small in comparison 
with the carrying capacity, one could treat $K$ to be a constant for all practical purposes. As Daly [24] argues, rapid increase in human population no longer allows us to consider our environmental resources to be infinite. Therefore, if not for ethical reasons, at least for reasons of practicality, the environment must factor into every discussion on sustainability. Current global consumption patterns point to a declining trend [25] in our natural resources. That this is a continuing trend is often contested by the argument that humans are resourceful and newer sources of energy and human capital will be discovered [26,27]. Daly [24] counters this argument by stating that: “... technology and infinite substitution mean only that one form of low entropy matter/energy is substituted for another, within a finite and diminishing set of low-entropy sources. Such substitution is often very advantageous, but we never substitute high entropy waste for low entropy resources in net terms".

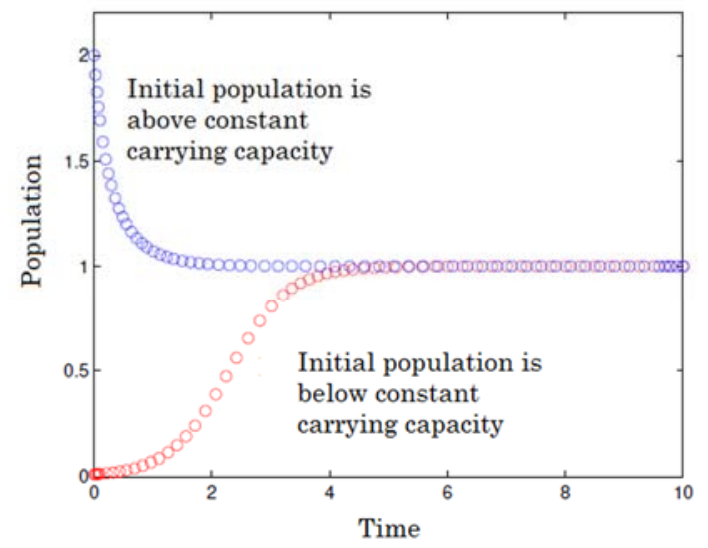

(a)

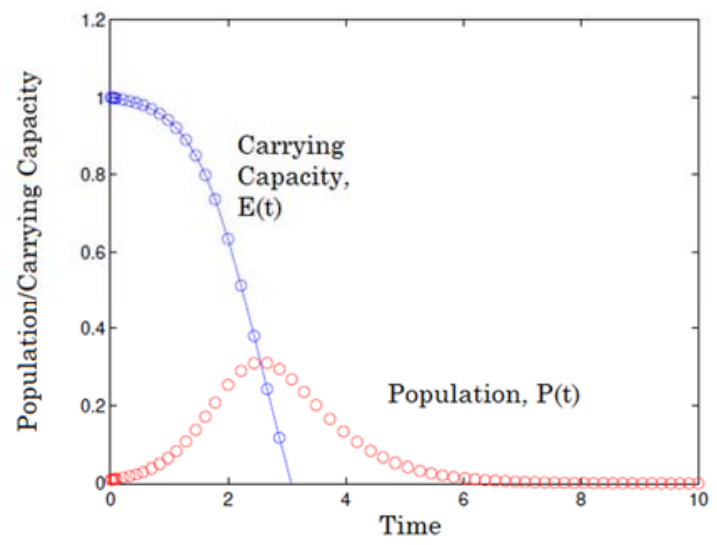

(b)

Figure 1. The above figures show the time evolution of population under the assumptions of (a) a constant and (b) diminishing carrying capacity. In the first panel, the population is constrained to reach an equilibrium value which is set by the constant carrying capacity, i.e., assuming that one can maintain a steady state of resources. When the population is sufficiently high, our high rate of consumption forces resources (carrying capacity) to decline with time which then drags down the population of the species with it.

Equation (1) is admittedly a simplistic way of discussing a very complex ecological system. However, the inclusion of all the ecological parameters in the model would make for a more urgent case for reduced consumption and a life of simplicity. Even a simple variant of this model where the population and resources are coupled shows that the ecosystem hangs on a delicate balance of optimality.

Let us now consider a different model where the carrying capacity is no longer a constant but is depleted at a rate proportional to the population. Therefore, we can write this idea mathematically as:

$$
\begin{gathered}
\frac{d P}{d t}=r P(1-P / E) \\
\frac{d E}{d t}=-r P
\end{gathered}
$$

where $P$ refers to the population and $E$ to the time-dependent carrying capacity. The numerical solution to this system of equations, as shown in Figure $1 b$, indicates that the situation is hopeless. Increasing population will deplete resources, which in turn very quickly erodes populations. Although the qualitative decay depends on where one starts, i.e., the initial value $P_{0}$, the result is qualitatively the same: eventual rapid demise! Such an observation is not new, nor are the model Equations (4) and (5) very sophisticated. More detailed models such as the Limits to Growth (LTG) study [28] have predicted 
similar consequences using detailed numerical simulations. In fact, recent updates to this modeling effort [29] and comparisons with population, economic, environmental data, all reveal remarkable fit to the model predictions. Figure $1 \mathrm{~b}$ shows a similar profile to the ones in the LTG models, where the population overshoot is followed by a dramatic downturn and collapse, accompanied by a rapid decline in resources.

Supporters of the growth models of economy argue that new technologies are constantly being invented to replace the outdated ones and these have historically shown to alleviate past social problems. However, what the mathematical models indicate and what is often forgotten (or conveniently neglected) is summarized by the well-known Jevon's paradox [30] (p. 94): “... new modes of economy will lead to an increase in consumption ..." While the environment is very resilient as a whole, sufficiently large shifts away from optimality can throw the existing balance into total disarray, as indicated in Figure 1b. The lessons of mathematics presented here, although simplistic in the social context, are significant. The "unreasonable effectiveness of mathematics" in describing physical phenomena is a puzzle to scientists as well, but there is little doubt about the power of mathematical modeling and analysis [31].

We mention here that simplicity and sustainability, from the mathematical perspective, evoke ideas of "constancy" (not to be interpreted as status-quo), as a pattern without fluctuations. In the language of mathematics, such a state of existence is described by the term "steady state". Laszlo [7] (p. 32) says: "The particular configuration of parts and relationships which is maintained in a self-maintaining and repairing system is called a 'steady state'. It is a state in which energies are continually used to maintain the relationship of the parts and keep them from collapsing in decay. This is a dynamic state and not a dead and inert one". Furthermore, in the words of Goldsmith [32] (p. 40):

"When a society becomes unstable, when social control breaks down and discontinuities grow even bigger, then it is but a question of time before it eventually collapses. It is towards such a collapse that our educational system, together with the rest of the institutions of our industrial society, are leading us. To avoid it, education must, among other things, be designed to promote stability rather than change-but this cannot be done in an industrial society in which the promotion of instability, implicit as it is in our notion of progress, is the avowed object of public policy".

In more rigorous mathematical terms, steady state is the eventual disappearance of temporal effects from a spatio-temporal system. Systems that reach steady state are said to have reached a state of equilibrium (with respect to their surroundings). The steady state condition also has a parallel in thermodynamics: closed dissipative systems in their steady state are said to have reached their maximum entropy production [33]. A steady state situation is often confused with the status-quo and its virtues are easily passed over in favor of growth. The value of a steady state condition in the context of economics was extolled even back in the 19th century by John Stuart Mill [34] (IV.6.9):

"It is scarcely necessary to remark that a stationary condition of capital and population implies no stationary state of human improvement. There would be as much scope as ever for all kinds of mental culture, and moral and social progress; as much room for improving the Art of Living and much more likelihood of its being improved, when minds cease to be engrossed by the art of getting on".

Muller [35] (p. 105) also mentions that: "When our political and ecological leaders urge us to 'save energy,' what they really mean is for us to generate as little additional entropy as possible. Entropy production implies energy has been 'wasted'; it has flowed from hot to cold without producing useful piston-pushing work".

At the heart of the steady state condition lies the idea of energy consumption which is rooted in the "immutable" 2nd law of thermodynamics [36]. As stated in Section 2, this law states that work necessarily produces waste. This loss of energy cannot be recovered and is essentially lost to the surroundings. This fundamental law of physics amounts to saying that, while we are destined to produce entropy by the very virtue of our existence, our lives are better spent trying to improve the quality of work performed corresponding to the same entropy production. There are no easy 
guidelines on which actions to perform and which actions will have direct and severe consequence on nature. A basic and minimum principle we can adopt is the Leoplod's maxim [37]: "A thing is right when it tends to preserve the integrity, stability, and beauty of the biotic community. It is wrong when it tends otherwise". A great deal of mathematical modeling relies on the hope or the expectation that: (i) we can understand; (ii) we can predict; and (iii) eventually we can control things or nature. However, as Systems Theory has bitterly shown us, this is not the case, in general, and other than very specialized cases, we rarely can predict or control things [6] (p. 168).

\section{Simplicity}

Simplicity is a concept with many meanings depending on the context and the situation. In this section, we examine various contexts where simplicity reveals itself with the aim of elucidating its role in supporting the goals of sustainability. Our initial hypothesis is that understanding, accepting and enacting simplicity in our daily lives is key to achieving sustainability in the long run in all spheres of human activity. Let us look at a few rudimentary definitions of simplicity.

\subsection{Functional Simplicity}

In everyday experience, simplicity evokes ideas related to lifestyles, functionality and usage, which is related to the utility of mundane objects. The use of basic objects, such as cups, spoons, fans, paper, pencil etc., is easily accepted without much thought. This is perhaps because the end function of the object in question is easily described and understood and has little to do with what goes into the inception, creation, and production of the object. No one would argue against the fact that the production of a seemingly simple object such as a spoon or a cup is very complex and requires detailed and expensive engineering applications which has little to do with its purpose or its retail price. In a recent lecture on this topic, chemist George Whitesides [38] breaks down the essential characteristics of simple objects, as possessing the following features:

FS1. Predictability, reliability

FS2. Cheap in terms of economic cost and material content of the product

FS3. Having value or functionality

FS4. Stackable: Can be used to create newer, more complex objects with different functions

The first trait FS1 tells us that the object can always be expected to perform what it is expected to do; there are no unintended outcomes. The property FS1 is related to FS3, namely that the object has an easily identifiable function but goes beyond it in requiring the object to consistently perform this one task. The cost effectiveness of the object (i.e., FS2) allows it to be configured in various ways to create new objects which can perform different and higher level functions (FS4). Whitesides offers the example of integrated circuits which have a stable function, are cheap and form the basic units of more complex devices such as cell phones.

Elaborating on the last point above, Whitesides states: "... the point is that [an] extraordinary simple idea rests on layers of simplicity each compounded into a complexity that is itself simple, in the sense that it is completely reliable ...".

Note that in defining objects put together in a complex manner as simple, based solely on its end-purpose, we are ignoring its history, the effort and the energy that went into its discovery and production. By this definition, complexity and simplicity become a function of time; therefore, what is complex at some time may become relatively simple at a later point, but never vice versa, since the property of "stackability" is uni-directionally defined. We suggest that an appropriate definition of simplicity (and complexity) should be independent of time, i.e., it should remain fixed in time. At a fundamental level, it is these rapidly changing attitudes which could be contributing to our overconsumption and environmental complacency. What one generation or a society defines as "a simple object" or "simple living" might be very different from a different generation or a different society in the same generation. Often there is a temporal or cultural context to this stacking and a 
lack of awareness of the simpler, underlying unit(s) that preceded the advent of the more complex object/function/practice. For instance, a bicycle is a very simple object in several wealthy Western societies but might be considered a luxury, even to this day, in very poor nations. Along the same lines, what is considered a simple life in the developed world might be extravagant in many developing societies. What we are suggesting here is a possible standardization of our definition of simplicity to bring about awareness of changes to some of the initial definitions of simplicity.

\subsection{Aesthetics and the Occam's Razor}

The idea of simplicity is also known in science and philosophy, to some extent, as the Occam's Razor Principle (ORP) or the Principle of Parsimony (PP) and essentially states that "Entities are not to be multiplied beyond necessity" [39] or in Isaac Newton's [40] words: "We are to admit no more causes of natural things than such as are both true and sufficient to explain their appearances". Furthermore, Post [41], providing a Popperian interpretation of simplicity, states:

"A theory is the simpler, the more easily it could be falsified. The degree of falsifiability depends on the number of parameters that have to be fixed in order to make it logically possible for the theory to be falsified. The simpler theory is richer, more powerful, it says more".

The concept of simplicity as used in science also has an aesthetic appeal, and a long-standing connection to the notion of "beauty". This has served as an important criterion in the promotion of scientific hypotheses to the stature of "theories". Since the time of Aristotle, it is a given that scientific theories are bound by this principle of beauty. Through this connection to beauty, aesthetics and efficiency have been brought under the same umbrella. Plato and Meskin [42] define "aesthetic value" as "... the value that an object, event or state of affairs ... possesses in virtue of its capacity to elicit pleasure (positive value) or displeasure (negative value) when appreciated or experienced aesthetically".

The 20th Century mathematician Paul Erdos constantly sought out the "book proof", by which he referred to the most elegant and succinct proof of a theorem [43]. Mathematicians of the stature of G.H. Hardy and Roger Penrose have shared their commitment and faith in aesthetics. Among the prominent physicists of the 20th century, Paul Dirac is famously quoted as having said [44]: "It is more important to have beauty in one's equations than to have them fit experiments".

Physicists, in the context of research and in the classroom, are attuned to casually attributing beauty to certain theories over others. Therefore, the task of the scientist or mathematician is not merely the search for explanations, but, in fact, to find simple explanations. The use of the word "simple" here signifies both "efficient" and "beautiful". Despite the seemingly overwhelming support for this romantic idea, there is criticism for the scientific obsession with simplicity. In his book Truth or Beauty, David Orrell [45] argues that to some extent the scientific fascination with beauty and simplicity has lead science astray from truth. He states [45] (p. 234): “... models have become confused with reality ..." However, these criticisms are few and far between and are directed mainly at science's perceived inability to resolve some long-standing fundamental questions. We therefore propose the following necessary condition for simplicity from a scientific perspective:

SS1: Given two or more choices, the one involving the least number of assumptions or hypothesis is the simpler one.

SS2: Given two or more choices, the one with the greatest aesthetic value can be considered the simplest choice.

\subsection{Personal Simplicity}

The suggested definitions FS1-FS4, SS1, SS2 can be adapted to provide traits of simplicity for an individual, i.e., a personal definition of simplicity. Accordingly, simplicity in a person or the actions taken by the person may indicate the presence of one or all of the following traits, not all of which are completely independent: 
PS1. Reliability and stability

PS2. Ability to live meagerly

PS3. Ability to live creatively and finding value in work and life

PS4. Living ethically

In fact, there seems to be a one-to-one mapping, i.e., a direct correspondence, between the traits FS and PS. In attempting to study an intangible concept such as simplicity, we find ourselves in the grips of several other intangibles which are directly or indirectly related to the core traits mentioned above. These might include: satisfaction, ethical or moral stability, wisdom and creativity. Each of these terms has been treated by moral and social philosophers, Eastern and Western [46,47]. In the following paragraphs, we look at some of these ideas. For example, Elgin [46] (1981, p. 117), in his pioneering book, Voluntary Simplicity, says: "Also found in the Hindu tradition is the idea of aparigraha or 'greedlessness' and 'nonpossessiveness'. It means to take only what we need and to find satisfaction in that".

The trait PS2 is perhaps the most widely discussed trait of simplicity in modern time, although perhaps misunderstood and/or misinterpreted. Simplicity very often evokes impressions of an outdated culture, mostly reserved for heretics or monastics. It is sometimes conflated with material poverty and as having little to do with or no interaction with our modern technological civilization. However, as Elgin [46] (1981, p. 34) points out:

"Poverty is involuntary whereas simplicity is consciously chosen. Poverty is repressive; simplicity is liberating. Poverty generates a sense of helplessness, passivity, and despair; simplicity fosters personal empowerment, creativity, and a sense of ever present opportunity. Poverty is mean and degrading to the human spirit; simplicity has both beauty and a functional integrity that elevate our lives. Poverty is debilitating; simplicity is enabling".

As in the case of functional simplicity, individuals need to be reliable, trustworthy and committed to their convictions. Many of the successes of industrial civilization, as exemplified by science and technology and the information sciences have injected an infinite growth mentality into our civilization. The ability to live meagerly refers to the degree of our reliance on external objects and also on the complexity of those objects. This brings us to the intermediate level technologies. Development of technologies that can mitigate the complexity of the objects while still preserving their essential functions can help draw us towards a more meager way of living. In this regard, Georgescu-Roegen [48] states:

"By 'technology' let us denote a package of feasible recipes containing at least one such recipe for every commodity necessary for the maintenance of the funds involved. That is, a technology consists of a general system by which environmental matter-energy is used for various purposes. To be sure, no technology can produce its own environmental source of support".

An appropriate or intermediate level of technology has been recommended by many, since it falls into the viable category. Georgescu-Roegen [48] indicates: "A viable technology is one that, just like a viable biological species, is capable of reproducing itself". The idea of appropriate or intermediate technology was emphasized by E.F. Schumacher [49,50] who said [50] (p. 95):

"In matters of development there is a problem of choosing the right 'level of technology' to fit the given circumstances; in other words, there is a choice of technology and it cannot be assumed that the level of technology used by affluent societies is the only possible level, let alone that it is necessarily the best for poor societies ... The technologies most likely to be appropriate for development in conditions of poverty would be in a sense 'intermediate' between — to speak symbolically — the hoe and the tractor, or the panga and the combine harvester".

Therefore, the ability to maintain a sense of stability (PS1) in this stream of rapidly changing scientific, technological, economic and moral setting is essential. Related to this idea is the trait of 
creativity. This characteristic has been studied by psychologists especially in trying to understand how the human mind is capable of accomplishing feats that appear to cross the boundaries of assumed human potential [51,52]. Based on the work of several scholars in the field of creativity [53-59], we identify the ingredients of creativity to include the ability to: (i) connect ideas; (ii) see similarities and differences; (iii) be flexible; (iv) be unorthodox; (v) be motivated; (vi) be inquisitive; and (vii) question norms. Entrepreneurs naturally value this trait for the material benefits that the cultivation of such a trait promises. Educators study this trait to enhance the educational experience of students [60-62] and to help produce well-informed citizens. The educational contribution of creativity is unfortunately conflated with the entrepreneurial definition, sometimes even by educators, who cater to creativity through material and technological improvements alone. We should emphasize that imagination is not the same as creativity, although we think they are related. We need imagination to create, but just because we are very imaginative does not mean we are also creative. Imagination is the necessary condition for creativity. As Robinson and Aronica [63] (p. 67) say: "You could be imaginative all day long without anyone noticing it. But you would never say that someone was creative if that person never did anything. To be creative you actually have to do something". Medawar [64] (p. 57) points out:

"That 'creativity' is beyond analysis is a romantic illusion we must now outgrow. It cannot be learned perhaps, but it can certainly be encouraged and abetted. We can put ourselves in the way of having ideas, by reading and discussion and by acquiring the habit of reflection, guided by the familiar principle that we are not likely to find answers to questions not yet formulated in the mind".

In highlighting this trait in PS3, we aim to promote it in a slightly different sense. We argue that creativity is the ability to learn to adapt to the changing environments, needs and times. All ingredients of creativity described above are still valuable and can be utilized to an end that does not just promote material growth.

In his book titled "Why we work" [65], Schwartz outlines the true nature of work and the common myths surrounding work and its true meaning. He concludes [65] (p. 17): "It is people who see their work as a 'calling', who find it most satisfying. For them, work is one of the most important parts of life, they are pleased to be doing it, it is a vital part of their identity, they believe their work makes the world a better place, and they would encourage their friends and children to do this kind of work". This attitude to work is crucial to fostering simplicity. It is important to find meaning in the actual work that is to be done rather than the end products of the work-usually money or material benefits, which can encourage dubious choices and cause damage to the larger community and future generations [65]. A focus on the process over the outcome or the end-benefit also naturally leads one to the path of creativity and makes one more adaptable to changing times and environments.

\subsection{Environmental Simplicity}

Thus far, we have focused our attention on the definitions of simplicity as they apply to the individual. However, in considering an ethically viable definition of this term, we need to consider how simplicity applies to the "other", i.e., to the environment as a whole and to other (non-human) species. One of the earliest and most fundamental ethical theories, related to environmental issues, was put forward by Regan [66]. He states two important conditions that we think must be satisfied by all theories related to environmental ethics $[67,68]$. If either of these two conditions is not satisfied, then it is not a fully satisfactory theory. In a sense, Regan provides a set of "necessary conditions" for the applicability or viability of any ethical theory as related to environment. These two conditions are:

(1) "An environmental ethic must hold that there are nonhuman beings which have moral standing".

(2) "An environmental ethic must hold that the class of those beings which have moral standing includes but is larger than the class of conscious beings-that is, all conscious beings and some nonconscious beings must be held to have moral standing". 
If we take the position that we have "no" duties towards other beings, then it will be easier to be unethical. This indicates a need for and the inclusion of the concept of species in environmental ethics. Gunn [69] says: "An environmental ethic is nonspeciestist in that it values each living thing, and each type of living thing, for its own sake. Or rather, it recognizes the value of the whole nature, including land forms and living things, not just instrumentally but also intrinsically".

Taylor [70], in an interesting article, discusses inherent worth, by asking the following question: "What does it mean to regard an entity that has a good of its own as possessing inherent worth?" To answer this, he uses the following two principles- the principle of moral consideration, and the principle of intrinsic value:

(1) "According to the principle of moral consideration, wild living things are deserving of the concern and consideration of all moral agents simply in virtue of their being members of the Earth's community of life".

(2) "The principle of intrinsic value states that, regardless of what kind of entity it is in other respects, if it is a member of the Earth's community of life, realization of its good is something intrinsically valuable".

Another interesting concept is irreplaceability of materials or beings. Martin [71] investigated the following argument:

“(P1) $X$ is irreplaceable.

(C) Therefore, X ought to be preserved".

An environmentally conscientious and ethical definition of simplicity therefore seems to go with discarding an anthropocentric attitude to life. In discussing the life of Albert Schweitzer, Marshall and Poling [72] (p. 190) note:

"To the man who is truly ethical all life is sacred, including that which from the human point of view seems lower in the scale. He makes distinctions only as each case comes before him and under pressure of necessity, as, for example, when it falls to him to decide which of two lives he must sacrifice in order to preserve the other. But all through this series of decisions he is conscious of acting on subjective grounds and arbitrarily, and knows that he bears the responsibility for the life which is sacrificed".

\subsection{Attitudes towards Simplicity}

It is very difficult, as with most issues encountered in ethics, to define exactly what simplicity is; thus far we managed to hint at some of its constituents. It therefore appears easier to compare this concept with other, somewhat better understood ideas in ethics and moral philosophy. In the tradition of W. Berry [73], we allude to certain important attitudes that we can assume which are essential to a simple life. In a world of finite energy and resources (food, minerals, etc.), one can choose from the following possible attitudes:

(1) Attitude of respect and reverence for life as a whole. In this idealistic view, we recognize that we are part of nature and that there are many other species striving to live and realize their potentiality. The nature of existence is such that we need material food to survive, and therefore, in some ways we have to take something away from nature. However, we do that in an attitude of reverence and conservation; conservation in the sense that if we really do not need it, we would not take it. We leave it for other beings and for the future generations. This may be called the attitude of custodian-ship or guardian-ship.

(2) Attitude of respect along with the notion of reusing whatever can be reused. It is difficult to clearly separate this category from the first one, but this might seem to be a more pragmatic attitude with acceptance of consumption behavior. This attitude is also accompanied by a respect for conservation principles whereby we do not callously discard items after using them; instead we try to reuse whatever is possible. The concept of conservation or "not using" is not as important or vital as in the first category and the concept of utility begins to enter into our system of ethics. This attitude may be called the attitude of partnership, with some responsibility as a custodian. 
(3) Attitude of recycling or extreme pragmatism. At this point we have generally put the burden of un-ease on others. We no longer have to think: (i) whether we really need to use this thing; and (ii) whether we can reuse. Instead, we use it and not even think about the consequences (at least not that deeply), yet we are aware that we need to recycle. However, recycling is not free; it requires energy and other raw materials. This attitude may be called the attitude of partial ownership, with a bit of responsibility as a partner.

(4) Attitude of not-caring (or not-thinking). This has been the predominant attitude in the last few hundred years, and especially more during the last few decades. Here, Nature is basically for man's use. There is no inherent or intrinsic value in nature as a whole, or in any other life form. The concept of utility is perhaps at its peak here. This attitude may be called the attitude of full ownership with all the rights associated with it.

These attitudes are summed up by Berry [73] (p. 7) in his classification of a strip-miner and a farmer as:

"I conceive a strip-miner to be a model exploiter, and as a model nurturer I take the old-fashioned idea or ideal of a farmer. The exploiter is a specialist, an expert; the nurturer is not. The standard of the exploiter is efficiency; the standard of the nurturer is care. The exploiter's goal is money, profit; the nurturer's goal is health-his land's health, his own, his family's, his community's, his country's. Whereas the exploiter asks of a piece of land only how much and how quickly it can be made to produce, the nurturer asks the question that is much more complex and difficult: What is its carrying capacity? (That is: How much can be taken from it without diminishing it? What can it produce dependably for an infinite time?) The exploiter wishes to earn as much as possible by as little work as possible; the nurturer expects, certainly, to have a decent living from his work, but his characteristic wish is to work as well as possible. The competence of the exploiter is in organization; that of the nurturer is in order-a human order, that is, that accommodates itself both to other order and to mystery. The exploiter typically serves an institution or organization; the nurturer serves land, household, community, place. The exploiter thinks in terms of numbers, quantities, 'hard facts'; the nurturer in terms of character, condition, quality, kind".

Thus, while an explicit definition of simplicity cannot be provided, we can glean some of its properties by considering the above-mentioned attitudes to life. Clearly, Attitude (1) and perhaps even Attitude (2) lead us closer to developing the traits of simplicity. Our increasing awareness of man-made climate change and the complex set of imminent problems that it is expected to cause is an important factor that must be considered in our analysis. Talking about sustainability, Daly [24] (p. 57) remarks: "As long as human economy was infinitesimal relative to the natural world, then sources and sinks could be considered infinite, and therefore not scarce... The economy has gotten bigger, the ecosystem has not".

As we have seen, the discussion of "simplicity" has been mostly the domain of philosophers, religious scholars and to some extent environmentalists, although a collective discussion by these diverse groups has not been very prevalent. In the previous sections, we have tried to present these various viewpoints with the aim of possibly unifying them into one consistent definition of "simplicity" and "sustainability". As scientists and mathematicians, we think that a more objective definition of simplicity can be provided to eliminate some of the confusion which may have arisen from the evolution of these ideas. In Section 5, we use a simple mathematical formula to illustrate these ideas. Mathematical models, in addition to explaining things, sometimes can have the power of prediction which cannot be achieved via philosophical considerations alone. Therefore, where possible, cautious modeling can be highly beneficial.

\section{Relating Simplicity to Sustainability}

In mathematical modeling, involving differential or integral equations, the goal is to solve the equations subject to initial and boundary conditions. There is a degree of determinism in such modeling approaches. The question of existence of solutions is always an important and valid study. In addition, 
if these equations are non-linear, multiple solutions exist and the question of uniqueness of the solutions and the stability of the solutions become important issues to consider. In many situations, a small variation (perturbation) in one of the parameters appearing in the equations may cause significant changes to the solutions causing instabilities or blow-ups, resulting in chaotic behaviors. Mathematical modeling of materials will also include certain guidelines and principles which need to be followed [74]. Perhaps the simplest type of mathematical modeling is using algebraic equations to relate a quantity (which is unknown) to some other parameters (which can be known). This approach has found a great deal of application and interest, especially, in non-mathematical fields, such as art, sociology, psychology, etc., (see the four volumes edited by Newman [75], especially Volume 4).

We propose that while the notion of simplicity or any of its related concepts are very complex, yet, they can lend themselves to mathematical analysis, which can shed more light on some of its essential qualities. In an attempt to formulate a mathematical theory of aesthetics and ethics, mathematician George Birkhoff applied simple logic to propose mathematical definitions for concepts such as "aesthetic measure" and "ethical measure" [76,77]. His work on a systematic quantification of aesthetics resulted in the formula:

$$
M_{a}=O / C
$$

where $M_{a}$ is the aesthetic measure, $O$ refers to order in the system and $C$ to its complexity. In his later work in 1941, Birkhoff offered the simple ethical formula:

$$
M=G
$$

where $M$ is the ethical measure and $G$ is defined as the total good achieved. While this definition at the outset may appear to be naïve and over-simplistic, the several examples that Birkhoff managed to construct surprisingly revealed the powerful capacity of this method to capture the ethical core in complex situations allowing for, at the very least, a nuanced discussion of the specific issue. The best case for such an argument is made by Newman [75] (2000, Volume 4, p. 2184), who stated:

"... they are unconvincing but never tedious. In any case it may be argued that mathematicians should have a turn at examining the beautiful and the good; philosophers, theologians, writers on aesthetics and other experts have been probing these matters for more than 2000 years without making any notable advance".

With the notions of simplicity (PS1-PS4, SS1-SS2, and ES1-ES2), given in Section 3, it might be possible to construct a formula for simplicity, in the vein of Birkhoff. What Birkhoff does so effectively in his article is to provide a way of quantifying difficult concepts such as "ethics" and "aesthetics". Following his strategy closely, we are encouraged to suggest a similar measure for the concepts of "simplicity" and "sustainability". One notion of sustainability, in elementary terms, can be thought of as the means to prolong a way of life which depends on our rate of consumption. Therefore, to be more sustainable, one must decrease the rate of consumption (i.e., amount consumed/time) which can be accomplished by: (i) reducing the amount consumed in a given time compared with previous trends; or (ii) prolong the consumption time, i.e., consuming the same amount over longer periods of time. Approach (i) can be related to PS3, i.e., the ability to live meagerly which we define as a fundamental trait of simplicity. Therefore, the two concepts of simplicity and sustainability are intricately connected. Approach (ii) suggests that we extend the time of consumption while retaining the same lifestyle. However, this raises the question if one has enough time and with growing world population, if this is even possible.

To formulate a definition of simplicity, we need several subsidiary definitions. The first of these is the object class, $O$, which is defined as the set of all objects which can be attributed the value of simplicity. For instance, this set can contain material objects whose functional simplicity can be evaluated; scientific theories whose relative simplicity can be gauged; or lifestyle choices whose social simplicity can be examined. A second natural set to be defined is the value class, $V$, which is defined 
as the set of traits used to define the traits of simplicity of $O$. Therefore, the value class is defined specifically for the object class in question. If the cardinality of the set $V$ is $n$, we define the measure of simplicity of the object class as

$$
M_{O}=\sum_{i=1}^{n} \gamma_{i} m_{i}
$$

where $m_{i}$ refers to the measure of simplicity of each trait in the value class and $\gamma_{i}$ is a real valued constant which indicates the relative strength of that trait. This parameter can be less than zero, signifying a deficiency of this trait or the presence of negative traits. How to quantify these traits and what rubrics to adopt is a harder question and is considered later. In fact, Birkhoff provides some clues about how to consider quantifying such a measure. It must be said at the outset that the specific choice of rubric to be used is not consequential. As long as we are consistent in defining our metrics, the relative measure of simplicity between any two competing choices in an object class will become apparent. What is clear is that the higher the value of $M_{O}$, the higher the simplicity attribute of the object.

We are now able to discuss a measure for sustainability, $M_{S}$, for which we borrow the formula suggested by Birkhoff, with a slight modification. Accordingly, we write

$$
M_{S}=\frac{M_{O}}{C_{E}}
$$

Here, $C_{E}$ refers to complexity, which in our case accounts for the sum of all the environmental and the entropic costs of this object. This term is essentially meant to account for all the externalities of the object which must be accounted for if we want to perform a life-cycle like analysis. Therefore, a higher value of $M_{S}$ would make the object more sustainable. This can naturally occur if: (i) $M_{O}$ is high; and (ii) $C_{E}$ is low. The former cases suggest that an object which caters highly to simplicity and negligibly to complexity, is sustainable. The latter case, however, says that even if the object is lacking in the simplicity measure, it can be sustainable if its environmental costs are negligible. One could argue that the formula must be given in a more general form such as:

$$
M_{S}=f\left(\frac{M_{O}}{C_{E}}\right)
$$

where $f$ represents an appropriate function. For the time being, we use Equation (9) for our analysis. One significant connection that can be made here is that this function $f$ determines the rate of population and resource collapse discussed in Model II in Section 4. In the case of diminishing carrying capacity, both population and resources have been shown to eventually disappear. However, what is still in our control is the rate at which this decline happens. We suggest that choices and actions which are higher in simplicity and lower in complexity cater to a lifestyle of higher quality and will produce a lower decay rate.

We can find more about the true test of the metric through specific examples. A point to note in applying these formulae is that they are not absolute or unique but applied in a comparative sense to contrast the sustainability of two objects. As Popper [78] (p. 197) says: "Every serious test of a theory is an attempt to refute it. Testability is therefore the same as refutability, or falsifiability. And since we should call 'empirical' or 'scientific' only such theories as can be empirically tested, we may conclude that it is the possibility of an empirical refutation which distinguishes empirical or scientific theories".

Example 1. Let us contrast two choices: walking and biking, on their measure of sustainability and simplicity. These are fundamental examples which are relatively easy to illustrate in an introductory article such as this. These examples can be extended to more sophisticated and complex objects such as electric cars or flying; however, we feel the details would take away from the point that we are trying to make. In addition, walking and biking are standard means of transportation in many countries. 
Object (or Choice) 1: walking as a means of transport, Object (or Choice) 2: bicycle as a means of transport. Therefore:

$$
O_{1}=\{\text { walking }\} ; O_{2}=\{\text { bicycling }\} ;
$$

Applying the properties FS1-FS4 to these objects, we see that

$$
V_{1}=\{\text { reliable, cheap }\} ; V_{2}=\{\text { reliable, cheap, functional, stackable }\} ;
$$

Then,

$$
\begin{gathered}
M_{O}\langle 1\rangle=\gamma_{1 r} m_{1 r}+\gamma_{1 c} m_{1 c}+\gamma_{1 f} m_{1 f} \gamma_{1 s} m_{1 s} \\
M_{O}{ }^{\langle 2\rangle}=\gamma_{2 r} m_{2 r}+\gamma_{2 c} m_{2 c}+\gamma_{2 f} m_{2 f}+\gamma_{2 s} m_{2 s}
\end{gathered}
$$

Here, the superscript corresponds to the measure for the $i$-th object. Since walking is a very elementary function, we do not see it as possessing high (or any) functional value compared to a bicycle, therefore $m_{1 f} \ll m_{2 f}$. What we mean here is that bicycling clearly has a functional advantage over walking, especially if one considers sufficiently long distances.

Similarly, under normal conditions, i.e., a healthy human being, clean air and safe roads, walking is cheaper and more beneficial than biking, therefore $m_{1 c}>m_{2 c}$. In terms of stacking, the bike has an advantage, therefore $m_{1 s}=0, m_{2 s}>0$, however, in the case of reliability, the human body is more reliable in a certain sense than a machine. Therefore, $m_{1 r}>m_{2 r}$. At the outset, walking appears to possess higher traits of simplicity than biking, although the latter has obvious advantages. However, the weighting factor $\gamma_{i}$ can skew the results in the other direction. This term is meant to account for "experienced value", desirability and feasibility of the object. Hence, for someone very poor, the impracticality of affording a bike outweighs any other decision, resulting in $\gamma_{1 f} \gg \gamma_{2 f}$.

Considering the complexity issue, the environmental cost of biking is much higher than that of walking, $C_{E}\langle 1\rangle \ll C_{E}\langle 2\rangle$. Therefore, it appears that in terms of the sustainability measure, $M_{S}{ }^{\langle 1\rangle}>M_{S}{ }^{\langle 2\rangle}$. In this case, walking is more beneficial than bicycling based on environmental considerations alone. In applying this analysis to practical life situations where we have to make a choice, for example, between walking and biking, the results of such an analysis, can point to a more sustainable and simple option. If the differences in the two measures are stark, then the choice to make based on this analysis is clear, otherwise, one could still go with the less favorable case.

The questions become most pertinent and difficult when we need to scale up from an individual to a population level, i.e., when we have to make a sustainable choice as a society. A trivial way of adapting Equation (9) would be to simply add a multiplicative factor in front of $M_{O}$ and $C_{E}$ that compounds the positive and negative effects appropriately according to the size of the population. This point needs further attention and will be addressed in greater depth in our future work.

The metric proposed here simply provides a basic justification for a choice; the final choice, however, is still rests in our hands and we can choose to ignore it. Naturally, we do not believe that, as a result of these metrics, cars will suddenly cease to exist and will be traded for bicycles, or even better, walking. As stated earlier in the paper, the definitions laid out here are independent of time. Therefore, just because our lifestyle [79] especially in Western societies is based or depends on using fossil fuel cars does not make it simple or sustainable. To consider the definitions time dependent, we could perhaps make the weight factors time dependent $\gamma_{i}=\gamma_{i}(t)$.

\section{Conclusions}

In opposition to the reductionist approach that the scientific and industrial enterprises have found useful and adopted for the past few centuries, the systems perspective has found a favorable voice in various branches of science since the beginning of the 20th century. The systems approach to understanding the world encourages a holistic approach, accounting for the big picture. As Laszlo [7] (p. 25) says: “"Wholes" and "heaps" are not mysterious metaphysical notions but clearly, 
even mathematically, definable states of complex entities. The decisive difference is that wholes are not the simple sum of their parts, and heaps are".

Systems theory provides the foundation to study the various possible interconnected pieces and interactions among various phenomena to describe certain observed patterns in nature [80]. The observation of these emerging patterns has made the idea of "wholes" more meaningful as a replacement of the classical reductionist view. The growing revelation of the underlying unity behind all-natural phenomena makes the idea of simplicity more appealing and compelling. As we have stated earlier, the fundamental laws of nature (thermodynamics) tell us that the creation and production entropy are the natural consequence of any action and proper accounting will reveal some form of waste, whether in the form of low or high entropy. Either way, it leads to the deterioration and collapse of the system, in finite time [29]. The underlying complexity of the human-environment interaction, we believe, calls for "simplicity" in our response to these complex problems. For instance, the solution to carbon emissions from fossil fuel-based vehicles could involve: (i) developing fossil fuel free vehicles such as electric cars; or (ii) redesigning entire cities and reconsidering urban development in such a manner such that individual vehicle ownership is no longer required. The former is a complex solution, the kind that we are aware of and willing to do. However, these approaches can only provide a temporary solution to our problems at best while the latter, we contend, is a simpler and more sustainable solution. It is agreed that urban redesign on a large scale and forgoing private material possessions are no easy tasks; however, the alternatives are far too dire for us to hold on stubbornly. Meadows et al. [28,29] argue that, among other things, in going forward, social and cultural changes on a massive scale are essential to achieving a sustainable future. Solutions to complex problems of the future caused by our past missteps and negligence requires creative solutions, but at the heart of it seems to lie simplicity.

It seems that all approaches to sustainability converge to the essence of simplicity: quality supersedes quantity, that sustenance is more practical, significant and even enjoyable in the long term than growth, which in time could stress the system to its breaking point. Living in a closed, finite universe where we have a finite amount of material resources, we can propose the following environmental norms (EN):

EN1: When we take anything which is more than what we really need (more than our share), we are depriving other beings, somewhere and at some time, of having the same or at least equal access to those resources.

EN2: Simplicity, in the modern age, and as a collective idea must include the suspension of growth as an economic idea but as an improvement in quality for all. From a social standpoint simplicity is therefore the most essential factor in sustainability.

In science, especially in mathematics and mathematical physics, simplicity and the search for elegant solutions is not the abandonment of rationality or scientific ideals. In the interest of reducing redundancy and obtaining a holistic understanding of the world around us, a simpler explanation, that makes the least number of detours (or assumptions) is naturally a preferable one and should be one of the initial goals of any scientific study. In the same vein, one of the eventual social goal needs to be the adoption of sustenance strategies over constant growth. A deeper conversation about the multifarious and unanimous definitions of simplicity must be encouraged; furthermore, the idea that simplicity is far removed from poverty must be realized. The achievement of simplicity does not signal the end of the civilization, as many might suggest. Simplicity favors relinquishing a one-dimensional economic view of the world for a more wholistic one which values the intangible elements of life as well. In the words of Daly [24] (p. 69): sustainable development is simply "development without growth".

In a television interview with Bill Moyers, Wendell Berry states: "To make a living is not to make a killing, it's to have enough" [81]. The concept of "enough" is what we define as "simplicity". In this paper, we have focused on why "enough" is both necessary and sufficient. The lessons of thermodynamics (energy and entropy) inform and warn us of the irreversibilties of many of 
our actions, whose negative repercussions can easily overtake any positives one might draw from over-production and over-consumption. While we have considered and discussed the theoretical side of "simplicity" and "sustainability", we offer some comments on the practicality and feasibility of such recommendations, keeping in mind that appropriate solutions can only be arrived at by being mindful and critically analyzing the feedback between experimentation and reevaluation. Some of the ideas we propose here are commonplace and widely espoused [82] though not often practiced. On a smaller, individual scale, this would amount to: optimal consumption of food and energy, reducing waste, using public transportation, recycling, refraining from using plastic bags and bottles, cooking one's own food, eating seasonal foods, etc. It becomes necessary to become mindful of one's own carbon footprint and redesign one's own life and habits of consumption to minimize negative impacts. On a larger scale, the cities and nations need to strive to adopt policies that de-emphasize an exclusive growth mentality and attempt to achieve sustainability; accumulation of wealth need not be the sole goal of individuals. Instead, we can pursue more meaningful goals and living creative and harmonious lives with respect for the biosphere.

In the 1950s, Norbert Wiener spoke out against the rapid development of automation and its potential negative impact on humanity [83]:

"It is perfectly clear that [automation] will produce an unemployment situation, in comparison with which ... the depression of the [nineteen] thirties will seem a pleasant joke. This depression will ruin many industries_possibly even the industries which have taken advantage of the new potentialities ...".

Such warnings have been made by a few scientists and philosophers, although they appear to have little impact on the rapid pace of growth of automation, artificial intelligence and information. Generally, proponents of these ideas are often dismissed as "naïve idealists". However, in the spirit of intellectual dialogue, we hope these ideas would remain on the table, and the future generations will have the option of considering and accepting some of the ideas advocated by the ideals of "simple and sustainable living." Therefore, what we, as individuals, can do is to continue striving for these ideals.

Author Contributions: M.M. and A.V. developed, discussed and co-wrote the article.

Conflicts of Interest: The authors declare no conflict of interest.

\section{References}

1. Daly, H.; Cobb, J. For the Common Good; Beacon Press: Boston, MA, USA, 1989.

2. Abdallah, S.; Thompson, S.; Michaelson, J.; Marks, N.; Steuer, N. The Happy Planet Index 2.0: Why Good Lives Don't Have to Cost the Earth; The New Economics Foundation (NEF): London, UK, 2009.

3. Helliwell, J.; Layard, R.; Sachs, J. World Happiness Report; the Earth Institute, Columbia University: New York, NY, USA, 2012.

4. United Nations Website. Available online: http://www.un.org/sustainabledevelopment/sustainable-deve lopment-goals/ (accessed on 29 November 2017).

5. Mainzer, K. Thinking in Complexity, 4th ed.; Springer: Berlin, Germany, 2004.

6. Meadows, D.H. Thinking in Systems; Chelsea Green Publishing: Hartford, VT, USA, 2008.

7. Laszlo, E. The Systems View of the World, 2nd ed.; Hampton Pr: New York, NY, USA, 1972.

8. Macy, J. Mutual Causality in Buddhism and General Systems Theory: The Dharma of Natural Systems; SUNY Press: Buffalo, NY, USA, 1991.

9. Bohm, D. Unfolding Meaning; Routledge: London, UK, 1985.

10. Adkins, C.J. Equilibrium Thermodynamics, 3rd ed.; Cambridge University Press: New York, NY, USA, 1984.

11. Massoudi, M. A System Theory approach to Interfaith Dialogue. Intercult. Educ. 2006, 17, 421-437. [CrossRef]

12. Moran, M.J.; Shapiro, H.N. Fundamentals of Engineering Thermodynamics; John Wiley \& Sons, Inc.: New York, NY, USA, 1988.

13. Kirwan, A.D., Jr. Mother Nature's Two Laws: Ringmaster for Circus Earth; World Scientific Publishing: River Edge, NJ, USA, 2000. 
14. Von Bertalanffy, L. General System Theory: Foundations, Developments, Applications, revised ed.; George Braziller Inc.: New York, NY, USA, 1968.

15. Kestin, J. A Course in Thermodynamics; Revised Printing; Hemisphere Publishing Company: Washington, DC, USA, 1979.

16. Kestin, J.; Dorfman, J.R. A Course in Statistical Thermodynamics; Academic Press Inc.: New York, NY, USA, 1971.

17. Georgescu-Roegen, N. The Entropy Law and the Economic Process; Harvard University Press: Cambridge, MA, USA, 1971.

18. Lindsay, R.B. Entropy consumption and values in physical science. Am. Sci. 1959, 47, 678-696.

19. Rifkin, J. Entropy, Into the Greenhouse World, revised ed.; Bentam Books: New York, NY, USA, 1989.

20. Massoudi, M. A Possible Ethical Imperative Based on the Entropy Law. Entropy 2016, 18, 389. [CrossRef]

21. Aris, R. Mathematical Modeling Techniques; Dover Publications, Inc.: New York, NY, USA, 1994.

22. Truesdell, C. An Idiot's Fugitive Essays on Science; Springer: New York, NY, USA, 1984.

23. Mazumdar, J. An Introduction to Mathematical Physiology and Biology; Cambridge University Press: New York, NY, USA, 1999; Volume 15.

24. Daly, H. Beyond Growth: The Economics of Sustainable Development; Beacon Press: Boston, MA, USA, 1996.

25. Pimentel, D.; Houser, J.; Preiss, E.; White, O.; Fang, H.; Mesnick, L.; Alpert, S. Water resources: Agriculture, the environment, and society. BioScience 1997, 47, 97-106. [CrossRef]

26. Lomberg, B. The Skeptical Environmentalist; Cambridge University Press: New York, NY, USA, 2001.

27. Simon, J. The Ultimate Resource 2, revised ed.; Princeton University Press: Princeton, NJ, USA, 1998.

28. Meadows, D.H.; Meadows, D.L.; Randers, J.; Behrens, W.W. The Limits to Growth. 1972. Available online: https: / books.google.com.hk/books?hl=zh-N\&lr=\&id=38tVDgAAQBAJ\&oi=fnd\&pg=PT25\&dq=+Mea dows, + D.H.\%3B+Meadows, + D.L.\%3B+Randers, +J.\%3B+Behrens, +W.W.+The+limits+to+growth\&ots=k otO9bZXcE\&sig=ZAaNF0npFB-xKKe-3xS5QDobBd8\&redir_esc=y\#v=onepage\&q\&f=false (accessed on 4 April 2018).

29. Meadows, D.; Jorgen, R.; Dennis, M. Limits to Growth: The 30-Year Update; Chelsea Green Publishing: Hartford, VT, USA, 2004.

30. Foster, J.B. Ecology against Capitalism; NYU Press: New York, NY, USA, 2002.

31. Wigner, E.P. The unreasonable effectiveness of mathematics in the natural sciences. Richard courant lecture in mathematical sciences delivered at New York University, May 11, 1959. Commun. Pure Appl. Math. 1960, 13, 1-14. [CrossRef]

32. Goldsmith, E. The Great U-Turn; Green Books: Devon, UK, 1988.

33. Chung, B.J.; McDermid, K.; Vaidya, A. On the affordances of the MaxEP principle. Eur. Phys. J. B 2014, 87, 20. [CrossRef]

34. Mill, J.S. Of the Stationary State. Principles of Political Economy Book IV: Influence of the Progress of Society; Longman Green and Co.: London, UK, 1848.

35. Muller, R.A. Now: The Physics of Time; W. W. Norton \& Company: New York, NY, USA, 2016.

36. Callen, H.B. Thermodynamics and an Introduction to Thermostatistics, 2nd ed.; John Wiley \& Sons: New York, NY, USA, 1985.

37. Leoplod, A. A Sand County Almanac; Oxford University Press: New York, NY, USA, 1949.

38. Whitesides, G. George Whitesides: Toward a Science of Simplicity; TED: New York, NY, USA, 2010.

39. Baker, A. Simplicity; The Stanford Encyclopedia of Philosophy: Stanford, CA, USA, 2016.

40. Newton, I. The Mathematical Principles of Natural Philosophy (Principia Mathematica); Citadel Press: New York, NY, USA, 1964.

41. Post, H.R. Simplicity in Scientific Theories. Br. J. Philos. Sci. 1960, 11, 32-41. [CrossRef]

42. Plato, L.; Meskin, A. Aesthetic value. In Encyclopedia of Quality of Life and Well-Being Research; Springer: Dordrecht, The Netherlands, 2014; pp. 76-78.

43. Hoffman, P. The Man Who Loved Only Numbers; Hyperioncop: New York, NY, USA, 1998; p. 26.

44. Koestler, A. The Act of Creation; Penguin Books: New York, NY, USA, 1964.

45. Orrell, D. Truth or Beauty: Science and the Quest for Order; Yale University Press: New Haven, CT, USA, 2012.

46. Elgin, D. Voluntary Simplicity; Harper Collins: New York, NY, USA, 1981.

47. Johnson, L.E. A Morally Deep World; Cambridge University Press: New York, NY, USA, 1991.

48. Georgescu-Roegen, N. Technology Assessments: The Case of the Direct Use of Solar Energy. Atl. Econ. J. 1978, 4, 15-21. [CrossRef] 
49. Schumacher, E.F. Small Is Beautiful; Harper \& Row: New York, NY, USA, 1973.

50. Schumacher, E.F. Good Works; Harper \& Row: New York, NY, USA, 1977.

51. Sternberg Robert, J. (Ed.) Handbook of Creativity; Cambridge University Press: New York, NY, USA, 1999.

52. Getzels, J.W.; Taylor, I.A. (Eds.) Perspectives in Creativity; Aldine: Chicago, IL, USA, 1975.

53. Sternberg, R.J. What is the common thread of creativity? Its dialectical relation to intelligence and wisdom. Am. Psychol. 2001, 56, 360-362. [CrossRef] [PubMed]

54. Neumann, C.J. Fostering creativity: A model for developing a culture of collective creativity in science. EMBO Rep. 2007, 8, 202-206. [CrossRef] [PubMed]

55. Spendlove, D. Creativity in Education: A review. Des. Technol. Educ. Int. J. 2005, 10, 9-18.

56. Getzels, J.W.; Csikszentmihalyi, M. The Creative Vision: A Longitudinal Study of Problem Solving in Art; Wiley: New York, NY, USA, 1976.

57. Simonton, D. Origins of Genius; Cambridge Press: New York, NY, USA, 1999.

58. Simonton, D.K. Genius, Creativity and Leadership: Historiometric Enquiries; Harvard University Press: Cambridge, MA, USA, 1984.

59. Simonton, D.K. Creativity. Cognitive, personal, developmental, and social aspects. Am. Psychol. 2000, 55, 151-158. [CrossRef] [PubMed]

60. Massoudi, M. Can scientific writing be creative? J. Sci. Educ. Technol. 2003, 12, 115-128. [CrossRef]

61. Munakata, M.; Vaidya, A. Fostering Creativity through Personalized Education. Primus 2013, 23, $764-775$. [CrossRef]

62. Munakata, M.; Vaidya, A. Encouraging creativity in mathematics and science through photography. Teach. Math. Appl. Int. J. IMA 2012, 31, 121-132. [CrossRef]

63. Robinson, K.; Aronica, L. The Element: How Finding Your Passion Changes Everything; Viking: New York, NY, USA, 2009.

64. Medawar, P.B. Induction and Intuition in Scientific Thought; American Philosophical Society: Philadelphia, PA, USA, 1969.

65. Schwartz, B. Why We Work; Simon and Schuster: New York, NY, USA, 2015.

66. Regan, T. The nature and possibility of an environmental ethic. Environ. Ethics 1981, 3, 19-34. [CrossRef]

67. Rolston, H., III. Environmental Ethics; Temple University Press: Philadelphia, PA, USA, 1988.

68. Singer, P. How Are We to Live; Prometheus Books: Amherst, NY, USA, 1995.

69. Gunn, A.S. Why should we care about rare species? Environ. Ethics 1980, 2, 17-37. [CrossRef]

70. Taylor, P.W. The ethics of respect for nature. Environ. Ethics 1981, 3, 197-218. [CrossRef]

71. Martin, J.N. The concept of irreplaceable. Environ. Ethics 1979, 1, 31-48. [CrossRef]

72. Marshall George, N.; David, P. Schweitzer: A Biography; JHU Press: Baltimore, MD, USA, 2000; p. 190.

73. Berry, W. The Unsettling of America; Sierra Club: San Francisco, CA, USA, 1977.

74. Truesdell, C.; Noll, W. The Non-Linear Field Theories of Mechanics; Springer: New York, NY, USA, 1992.

75. Newman, J.R. The World of Mathematics; Dover Publications: Mineola, NY, USA, 2000; Volumes 1-4.

76. Birkhoff, G.D. A mathematical theory of aesthetics. Rice Inst. Pam. 1932, 19, 189-342.

77. Birkhoff, G.D. A mathematical approach to ethics. Rice Inst. Pam.-Rice Univ. Stud. 1941, $28,23$.

78. Popper, K. Conjectures and Refutations, 5th ed.; Routledge: London, UK, 1989.

79. Naess, A. Ecology, Community, and Lifestyle; Cambridge University Press: New York, NY, USA, 1990.

80. Bar-Yam, Y. Dynamics of Complex Systems; Addison-Wesley: Reading, MA, USA, 1997; Volume 213.

81. Berry. Available online: http://billmoyers.com/segment/wendell-berry-on-his-hopes-for-humanity/ (accessed on 4 April 2018).

82. Alexander, S. Sufficiency Economy: Enough, For Everyone, Forever. Available online: http://simplicitycoll ective.com/enough-for-everyone-forever (accessed on 4 April 2018).

83. Wiener, N. The Human Use of Human Beings: Cybernetics and Society (No. 320); Perseus Books Group: New York, NY, USA, 1988.

(C) 2018 by the authors. Licensee MDPI, Basel, Switzerland. This article is an open access article distributed under the terms and conditions of the Creative Commons Attribution (CC BY) license (http:/ / creativecommons.org/licenses/by/4.0/). 